the opening chapter, where the weight of Maddison's annotation will defeat all but the most indefatigable. Elsewhere, however, one wishes for rather more: the book's scholarly usefulness would have been surely greater if references to correspondence printed by Birch had also indicated the manuscript source where this is extant. Equally one sometimes wishes for more extended treatments of particular aspects (especially that of the Invisible College). Maddison assumes a great deal of background information in the reader of what is, none the less, a basic work.

A. J. TURNER

\section{SOLVENT CHEMISTRY}

\section{Non-Aqueous Solvents}

By T. C. Waddington. (Studies in Modern Chemistry.) Pp. vii +88 . (Nelson: London, November 1969.) 40s boards; $20 s$ paper.

THE book is short, comprising six chapters covering solute-solvent interaction, protonic solvents, liquid oxide solvents, oxyhalide solvents, liquid halides and fused salts. The first chapter is deservedly the longest, dealing with the energetics and mechanisms of solvation and solvent self-ionization. In this field it is refreshing to find recognized a few non-aqueous solvents, for example, acetone, which are not noted for self-ionization or participation in reactions. It is also encouraging to find solvation differentiated from ligand coordination. All the solvents discussed are adequately documented by their physical data. Each chapter ends with a short bibliography and some revision exercises.

The sections on particular solvent typos are excellently furnished with explicit stochiometrical equations, which makes for smooth reading. The book is suitable as a rapid sketch of the field for a student, but some additional background explanation would improve it in this respect. Thus, for example, Lewis acids and the Lux-Flood theory are rather taken for granted. Similarly, while the electrochemical aspects of the various solvents are well recognized, apart from a reference to the Shedlovsky equation the theoretical bases of $\Lambda_{0}$ and $\mathrm{K}_{\mathrm{A}}$ are left undefined. On page 44 the author identifies chemical affinity with exothermicity and it is to be hoped that he will subsequently correct this general impression even if the case in point seems an approximate justification.

\section{S. R. C. Hughes}

\section{MODULAR FORMS}

\section{Modular Forms and Dirichlet Series}

By Andrew Ogg. (Mathematics Lecture Notes Series.) Pp. xviii +173 . (W. A. Benjamin: New York and Amsterdam, 1969.) n.p.

These notes cover the basic properties of Hecke operators on the modular group and its principal congruence subgroups, and of Dirichlet series with Euler products and functional equations. Chapter one is an account of Hecke's work on Dirichlet series with signature $(\lambda, k, C)$. Chapter two discusses Hecke operators on the full modular group, treated as correspondences on a set of lattices, completed in chapter three using the Petersson inner product. Chapter four extends the theory to congruence subgroups, including the explicit construction of Eisenstein series. Chapter five gives Weil's characterization of forms on $\Gamma(N)$ by means of functional equations for associated Dirichlet series, and chapter six gives a brief account of theta series based on quadratic forms following Hecke and Schoeneberg.

The book covers material not readily available elsewhere, because the literature is diffuse even for readers of
German. Hecke's own papers do not use the Petersson inner product, and Petersson's refer remorselessly back to Hecke for basic results, as well as being too general for a first reading. The author's style is discursive, with frequent digressions and anticipations, but this is an advantage for didactic purposes. A disadvantage for the novice is his cavalier treatment of the main definition: a "modular form" is defined three times (II-2, III-5, IV-3), each relevant for its immediate context, but the reader should be told that other writers might allow poles but disallow any subgroup of $G L^{+}(2, R)$. Similarly the author's definition of "dimension" agrees with those of Klein, Hecke and Petersson, but his notation ( $k$ for $-k$ ) does not; and "level" is not always confined to congruence subgroups. Finally, the author's convention (I-32 et seq.) that $-1 / \tau+1$ means $-1 /(\tau+1)$ really is peculiar to him. self, and he should warn the reader in advance. But these are minor issues which do not arise if the book is used as a text for a lecture course, and the author's treatment of the subject is largely both logical and readable.

It is therefore unfortunate that the presentation and appearance of the book do not do the text justice. The book was printed directly from a typescript, in the interests of rapid publication. The typing is neatly done; though inevitably not as clear as print when so many suffixes and indices are used, but there are some needless visual difficultios. The text runs on continuously, broken only by chapter headings and the underlining of definitive words, so that the main results do not stand out. It is often hard to see when the statement ends for a theorem proved in advance, or the proof for one proved in arrear. These faults, however, can largely be remedied with a pen and ruler, and do not detract seriously from a useful addition to the literature.

A. O. L. Atkry

\section{MATHEMATICAL LOGIC}

\section{Mathematical Logic and Hilbert's $\epsilon$-Symbol}

By A. C. Leisenring. (University Mathematical Series.) Pp. ix +142 . (Macdonald (Technical and Scientific): London, October 1969.) $60 s$.

THIs book on mathematical logic is very much devoted to Hilbert's $\varepsilon$-symbol, a subject that normally is not given prominence in textbooks. The author has tried to make the book self-contained and hopes that it will be useful as supplementary reading in an undergraduate course. The $\varepsilon$-symbol has some advantages in formal set theory; only time will tell whether it should have a higher status as a topic in mathematical logic-my opinion is that its status will not change very much.

The book is well written, but the reader will need to start with some background knowledge of logic. The content of the book is sufficiently distinctive for it to be recom. mended as supplementary reading to any course on logic, and the book would be a useful addition to any library.

The text begins with an eight page introduction that is readable and worth reading. The first chapter introduces a formal language and studies the semantics of this language. Deductive properties of systems based on the formal language are studied in the second chapter. Some knowledge of a standard text may be helpful to put in perspective the author's stress on the $\varepsilon$-calculus and its advantages over the predicate calculus. In chapter three Hilbert's $\varepsilon$-theorems (which say, very roughly, that the $\varepsilon$-calculus and predicate calculus are equally powerful) and some related results are proved, and in chapter four it is shown that the $\varepsilon$-calculus is useful in formal set theory and in proofs of consistency. In the last chapter, a Gentzen type system that incorporates the $\varepsilon$-symbol is introduced and a cut elimination theorem is proved.

The book is well produced and ends with a four page bibliography and eight pages of indexes.

R. M. DICKER 